
international learning. In Gómez Chova, L., López Martínez, A. and Candel Torres, I. (eds.) Proceedings of the 12th

International conference on education and new learning technologies (EDULEARN20), 6-7 July 2020, [virtual conference]. Valencia: International Academy of Technology, Education and Development (IATED) [online], pages 3564-3571. Available from: https://doi.org/10.21125/edulearn.2020.0997

\title{
Professional learning through collaborative online international learning.
}

SWARTZ, S., BARBOSA, B., CRAWFORD, I. and LUCK, S. 


\title{
PROFESSIONAL LEARNING THROUGH COLLABORATIVE ONLINE INTERNATIONAL LEARNING
}

\author{
S. Swartz' ${ }^{1}$ B. Barbosa ${ }^{2}$, I. Crawford ${ }^{3}$, S. Luck ${ }^{4}$ \\ ${ }^{1}$ Mainz University of Applied Sciences (GERMANY) \\ ${ }^{2}$ University of Aveiro (PORTUGAL) \\ ${ }^{3}$ Robert Gordon University (UNITED KINGDOM) \\ ${ }^{4}$ Pfeiffer University (UNITED STATES)
}

\begin{abstract}
Collaborative Online International Learning (COIL) presents relevant opportunities for innovation in the teaching-learning process [1] [2]. Global virtual teams (GVTs) activities provide a very broad set of advantages, including the development of soft skills. In addition to enhancing business communication skills, the learning outcomes from these activities indicate an increase in intercultural competencies, virtual teamwork and project management skills. COIL projects are helpful in achieving these goals while offering instructors the opportunity to develop as global educators. Thus, the benefits of COIL are not reserved for students, but rather positively impact instructors through the exchange with colleagues from diverse institutions, backgrounds, pedagogies and practices. Instructors thereby gain global understanding which they can impart to their students and use to help facilitate intercultural curricula at their institutions. Engaging in GVTs projects means intense collaboration and agreement between faculty on assignments, deadlines, assessment and learning outcomes. This, in turn, forces instructors to reevaluate their own values and methods of work. This paper describes several COIL projects which instructors from four universities in the USA and Europe carried out together with their students over the course of several years. These instructors increased their own professional learning experience by overcoming technological, institutional and cultural differences. It is the hope of this study to encourage faculty and colleagues to engage in and facilitate the use of COIL projects as part of a larger endeavor to internationalize higher education.
\end{abstract}

Keywords: collaborative online international learning; global virtual teams; global learning environments; professional learning; internationalization of higher education.

\section{INTRODUCTION}

While most educators agree that internationalization is a priority in the education of graduates, most institutions fail to establish it in a consistent, concerted effort across study programs and curricula. Despite proclamations to the contrary, efforts at internationalization at university campuses have been instead "piece-meal and reactive, rather than coherent and holistic" [3]. Internationalization is often delegated to international offices or centers for global experience. While sending students abroad is an important contribution towards encouraging global thinking amongst graduates, and the numbers of students attending a foreign university are rising, nevertheless, only a handful of students have the economic privilege to take advantage of this opportunity [4]. Efforts to incorporate global topics into curricula are sporadic and depend on individual faculty members and department chairs, who may (or may not) recognize the value of intercultural experience. At the same time, the efficacy of these efforts may vary based on the level of staff experience and insight [5]. In worst case, attempts at internationalization could actually result in the opposite of what was intended. Poorly designed initiatives might cause a lack of understanding and less desire among students to work with people of another culture.

The need for internationalizing the curricula of higher education institutions has received increased attention [3] [6]. In this context, educators are expected to agree on the international learning outcomes required for graduates to be successful in a global workplace. Furthermore, this process requires a systematic approach with clear learning outcomes and the development of "authentic tasks that are structured in such a way that they cannot be successfully completed without a meaningful exchange of cultural information" [7]. Leask and Bridge asserted that internationalization must address new approaches to curricula which are not grounded in predominantly Western notions of education [3]. Moreover, this intercultural curricular approach should go beyond the classroom and encompass 
all university activities both on and off campus [7]. Instructors should function as facilitators for intercultural understanding and mutual respect between students of different cultures [8].

The aforementioned approach to internationalization of institutions presumes that instructors have had considerable international exposure as well as possess intercultural competencies themselves. However, even those instructors who have taken part in staff mobility opportunities do not necessarily have an understanding of how to implement internationalization in their home contexts. High quality staff development training is essential for instructors in order to establish and assess internationalization efforts effectively at their institutions [6]. Moreover, internationalization at institutions of higher learning is not only the responsibility of instructors but must involve a concerted effort of all university stakeholders.

Introducing internationalization into classrooms requires new approaches to teaching, acquiring new skills, collaborating with external and internal partners as well as creating new global network learning environments (GNLEs) [9]. Faculty who are to be global educators and facilitators of change must meet high expectations. Not only do they have to acknowledge the importance of global understanding for themselves and their students. In addition, they have to reflect on their own perceptions, evaluate their accuracy in a changing world, and continually alter their behavior accordingly. At the same time, instructors are expected to guide their students through the same transformations in perception and behaviour that they themselves may be going through.

Collaborative Online International Learning (COIL) presents relevant opportunities for innovation in the teaching-learning process [1] [2]. Global virtual teams (GVTs) activities provide a very broad set of advantages, including the development of soft skills and increased motivation of students. In addition to enhancing business communication skills, the learning outcomes from these activities indicate an increase in intercultural competencies, virtual teamwork and project management skills. COIL projects are helpful in achieving these goals while offering instructors the opportunity to develop as global educators.

Thus, the benefits of COIL are not reserved for students, but rather positively impact instructors through the exchange with colleagues from diverse institutions, backgrounds, pedagogies and practices. Instructors thereby gain global understanding which they can impart to their students and use to help facilitate intercultural curricula at their institutions. Engaging in GVTs projects means intense collaboration and agreement between faculty on assignments, deadlines, assessment and learning outcomes. This, in turn, forces instructors to re-evaluate their own values and methods of work.

This study describes several COIL projects which instructors from four universities in the USA and Europe carried out together with their students over the course of several years. In addition to the developments of the projects, the learning process and the deliverables over time, the focus is on the challenges of creating a common team culture amongst the faculty members. Furthermore, these instructors increased their own professional learning experience by overcoming technological, institutional and cultural differences. By sharing their experiences, this study hopes to encourage faculty and colleagues to engage in and facilitate the use of COIL projects as part of a larger endeavour to internationalize higher education.

\section{METHODOLOGY}

In the following, the authors describe COIL projects which they carried out in their respective courses over the course of several semesters with numerous variations. First, the background provides information as to how their partnership came about and how it progressed over time. Furthermore, the projects themselves are described, including what kind of students were involved, how the assignments were set up and how the collaborative projects were assessed. Moreover, the motivations of the instructors and their learning outcomes for their students are discussed. Finally, the instructors' reports their mutually agreement on the benefits of the projects are illustrated.

\subsection{Background}

The groundwork for faculty collaboration began in 2015 when an instructor from the United States and a colleague, an American instructor teaching at a German university, met for lunch. They began discussing collaboration opportunities during which the idea to connect their Business Communication courses through a virtual team project was born. Excited about a new teaching challenge that they were sure would mutually benefit their students, they set out to create a project description and a 
timeline of six weeks which culminated in a teleconference involving both the American and the German students discussing their project deliverables. The experience was so positive that they continued creating such projects every semester with slight variations in the project topic. After several semesters they decided they would like to increase the scope of the project and bring additional cultures on board. A third instructor from Scotland responded to a general enquiry for interested colleagues sent out to international partner universities. After a presentation of the virtual team project at EDULEARN in Barcelona, the instructor from Germany met with a fourth colleague from Portugal, who was presenting her own COIL projects, and discussed future collaboration. After several skype meetings, together and individually, continuous emails and instant messages, the four instructors put together a concept for a collaborative project involving all four universities, which first took place in the fall of 2017 and has been repeated with variations every fall term since then.

\subsection{Project description}

\subsubsection{Participants}

The students involved in these intercultural collaborative projects differed from one another in more than their national culture; and disparities existed among the students in gender, ethnicity, generation, educational level, professional experience and family status, as well as areas of study and length of time at university. Students from the USA were enrolled in graduate programs in the areas of health and business administration; they were professionals seeking to further their careers and were taking part in either seated or online courses on organizational communication. The Americans were diverse in terms of ethnic as well as regional backgrounds, and many of them had families. The Scottish teams involved both seated and online students of corporate communication and public affairs and fashion management. They represented a range of cultural, ethnic, academic and professional backgrounds. The Scottish students were undertaking - both online and seated - a postgraduate module in public relations theory and practice as part of a wider MSc course of study. The German teams were made up of seated undergraduate students of business administration (sophomores) and applied computer sciences (freshmen). The business students were taking part in an elective which focused on working in multicultural teams and their English language abilities were advanced. A third of the class were foreign students visiting through an Erasmus program. The language abilities of the computer science students varied considerably and the majority were foreign students primarily from Northern Africa or raised in families of non-German ethnic origins. They were participating in a compulsory English communication course. The students from the Portuguese university were undergraduate, primarily seated students of marketing and were ethnically homogeneous, although a few of them were mobility students from other European countries. They were third-year students enrolled in the course Digital Marketing, which is particularly focused on creating strategies and producing content to interact with target audiences on the Internet.

\subsubsection{Assignments}

The initial COIL assignment involved American and German students representing Reebok and Adidas in a simulation of the real-life merger of the two sports giants. Students were assigned into teams and were asked to collaborate on topics involving human resource policies, corporate identity and data protection. Their ideas were discussed in a live teleconference and finalized in a memo to the board of directors.

The proceeding assignment involved investigating potential difficulties that companies experienced on the foreign market, such as ALDI or LIDL in the United States, HUGO BOSS in the UK or Walkers Shortbread in Germany. These difficulties could encompass brand recognition, human resource policies or competitors. Students were expected to analyze these difficulties with the help of modals such PESTLE or SWOT and agree on possible solutions. Their analyses as well as suggestions for changes were presented to the classroom; the other students functioning as a board of directors and ideally subjecting the presenters to critical questioning. In the case of the Portuguese and German teams, the assignment was adapted to better fit the syllabus and learning objectives of the Portuguese class. In this case, the teams developed ideas and marketing strategies to encourage a rise in tourism to Portugal and to make Germany more attractive in order to attract Portuguese professionals to fill needed positions in Germany. These assignments culminated in videos which were created by the Portuguese digital marketing students and supported by the German team members.

The most recent COIL project concerned analyzing the online presence of global operating companies. Students chose companies including Lufthansa, Starbucks and Google, and delved into 
their presence on Instagram, Facebook and other social media as well as their own websites. In particular, they took a look at their cultural appropriateness in the individual countries. The students presented their analysis and recommendations for improvement in the form of online Prezi presentations. They then gave their findings to their respective classes for discussion, and summed up their experiences in short reports.

\subsubsection{Use of technology}

The initial communication between teams occurred through email. Having once established contact, students arranged Skype or Zoom meetings (the project required three meetings) and agreed on the collaborative tools they intended to use. While instructors recommended the use of SLACK and Google Drive for file sharing and collaborative writing, students were relatively free to choose from all available platforms, social media as well as IM apps such as WhatsApp or Messenger. The universities utilized different learning management systems (i.e. the USA with Blackboard, Germany with OLAT) so it was not possible for instructors to arrange a collaborative space common to all.

\subsubsection{Workload and focus}

As was mentioned above, the project was either built-in to the course or added on depending on the requirements and schedules of the different university courses. The instructors chose $5-6$ week periods which suited each course schedule. Whereas the German students were given time during their seated courses to work with their partners on their projects, the online courses at the American university expected students to work outside their regular coursework and without seeing their local partners. This fact impacted the role of the instructor in terms of mentoring students and their progress. In classes which met in person once a week, students voiced concerns or pointed to glitches so that solutions could be found quickly, reducing frustration levels, whereas in other classroom contexts a considerable amount of time passed before instructors became aware of difficulties and could offer assistance.

\subsubsection{Student evaluations}

The instructors deliberately did not monitor student performance during the course of the projects. Students were expected to fulfill certain benchmarks at the beginning of the project, such as establishing contact and exchanging information about each other as well as designating roles and tasks, in order to ensure a relatively smooth flow. For most of the projects, the teams worked independently unless they requested guidance. Once the use of SLACK was introduced to the project, instructors joined the workspaces to be able to monitor progress and solve problems quickly if necessary.

The final paper and/or presentations or in some cases the videos were the focus of evaluations. Existing discrepancies in the weight of the assignments and in the marking criteria of the projects between the four participating courses led to differences in perceived project significance between the teams, which in turn impacted student performance. Once the instructors realized this, more effort was made to synchronize the value of the project towards the final grade as well as to align final grades after group assessment sessions.

\subsubsection{Project challenges}

The challenges the instructors faced were multifaceted: each university had different semester schedules and varying requirements for each course. They found a six-week slot that fit with a few slight adjustments (the Americans and the Scots began and ended the project a week earlier). Whereas the German, Portuguese and American students had the projects built-in or rather integrated into their course syllabus, the English students experienced it as an add-on. This impacted the latter students' willingness to engage in the project as did differences in the demands on and evaluation of students. When the Scottish students initially joined the project, their participation was voluntary; however, this was increased to at least $20 \%$ of their final grade. Students from Germany received $50 \%$ of their final grade related to the project.

As mentioned above, the teams were very heterogeneous. In addition to varying in demographics, the students also differed in their English language abilities, ranging from native speakers to intermediate command. Furthermore, the courses involved various disciplines within undergraduate and graduate business programs and were taught either online or seated. 


\subsubsection{Learning objectives}

The instructors shared a mutual understanding of the objectives of these global virtual teams collaborations. By facilitating COIL projects, they aimed at improving students' collaborative writing and speaking skills, intercultural competencies, teamwork skills, use of digital channels in cross-border communication and skills in communicating with individuals whose native language is different from the other team members. The project also focused on familiarizing students with common business communication practices as well as honing their project management skills. Developing students' intercultural sensitivity and intercultural communication competence while communicating and collaborating within international teams was an essential objective in the COIL projects previously described. As one instructor put it: "For me, reflecting COIL in the learning objectives is essential. Obviously it creates adequate expectations on the students, but it also shows the commitment to COIL (including mine). It poses an additional challenge, regarding measuring this outcome, which can be solved for instance with peer and self-assessment" (Portuguese instructor).

\section{RESULTS}

The four instructors carried out the projects in addition to their regular course load and administrative duties. Before the project even began, during and after the project, the instructors met virtually and communicated through email and the collaborative platform SLACK regularly over several months. None of the instructors were digital natives, and yet they were teaching students who placed value on virtual as well as experiential learning and were primarily concerned with how this knowledge offered them better career chances [10].

In order to address these concerns, the instructors needed to hone their own technical skills and not only provide instructions but also be prepared to demonstrate their understanding of digital communications software and collaborative platforms. Experimenting with creative software such as Powtoons, sharing ideas on SLACK and writing collaboratively on Google Docs was not only fun but also afforded the instructors a deeper understanding of the advantages as well as challenges and limitations of digital communication.

Since their learning management systems were incompatible, the instructors resorted to open source programs, and taught themselves as well as guided each other through the use of the technology. The assignment as well as benchmarks and expectations for the project developed out of trial and error from previous experiences and feedback from students. Moreover, deviations in semester schedules, holidays, curricula and workloads needed to be factored into the project. Whereas the instructors in continental Europe were fairly sovereign considering their syllabi, the Anglo-American instructors were more confined by department-held demands. Thus, any assignments students were expected to do for the project were in addition to mandatory papers and exams expected for course credit.

Assessing students in light of deviating grade values presented challenges. While instructors agreed on group grades, they could consider individual contributions through the SLACK workspace feeds and thus still maintained autonomy when evaluating their own students.

Data protection regulations in the European countries (GDPR) required careful consideration when sharing students' private data. Instructors both in and outside Europe were expected to have students sign consent forms in order to exchange student email information or to require their students' use of platforms such as SLACK. The instructors informed themselves about institutional as well as state policies in the different countries and followed procedures involving the gathering of data for research purposes required by their ethics boards. As a result of the issues mentioned above, the instructors found themselves working around the individual constraints of each member of the team in order to realize their COIL projects.

Throughout the project and afterwards, the instructors stayed in regular contact with each other so that meetings became a habit. While the project was the focal point, they often shared personal information such as vacations and family illnesses. Through video chat, they entered each other's living spaces while family members came in and out of view. This way, they developed close, personal relationships although most of them had not met all the colleagues face to face.

The personal relationships and familiarity with each other's pedagogy enabled trust to develop, which was fundamental to sharing course content, aligning syllabi and assessment criteria, even interacting with each other's students; in other words, the instructors trusted each other enough to give up autonomous control over their courses and to rely on other's judgement. This close interaction 
inevitably implied reflecting on own pedagogical approaches and assumptions on methodology and goals.

Furthermore, the insights which emerged during the course of the project impacted the instructors' pedagogy to the extent that course content was modified, topics were added, assignments were altered or changed completely, and new approaches were adopted. By familiarizing themselves with other disciplines and research areas, the instructors increased their pool of knowledge, from which their students and colleagues could profit.

An appreciation of each other's cultural differences arose through the exchange. Each instructor contributed their particular strengths to the team. One instructor's expertise in developing assessment criteria was utilized to create a common assessment rubric for the presentations and paper. As two members were interested in research, the instructors carried out project surveys to measure the development of intercultural competency among students.

The instructors delved into disciplines and areas of research previously less familiar to them. Investigating, sharing literature and discussing approaches was an exciting experience. In turn, they felt the need to share their ideas and research with colleagues outside their collaborative projects through international and national presentations, publications and through workshops at their own institutions. The input they received contributed towards refining both the COIL projects as well as their research.

During their COIL projects, the instructors shared their visions of the learning outcomes they intended for their students, agreed on the parameters of the projects, adopted mutual assessment criteria and found compromises to overcome individual faculty and institutional restrictions. They each contributed their individual strengths and took on or delegated tasks according to interest and in light of individual constraints. Thus, the collaborations described here created a "shared team culture ... vital to team success because it facilitates communication, coordination, and the sharing of knowledge, thus increasing team members' familiarity with each other's expertise, work habits, and local contextual constraints" [11].

In general, the instructors encountered little resistance on the part of their university heads to their COIL project endeavours. However, institutional practices regarding workload, budgets or use of IT infrastructure often presented themselves as constraints. The instructors frequently found themselves investing their own personal time, money and resources in order to facilitate the projects. Regrettable was the lack of funds to meet up face to face or attend conferences together. Often those colleagues with more financial support from their institutions carried out presentations on their projects and research while others participated virtually or not at all.

Nonetheless, the authors hope in future to gain institutional support and advance policy changes conducive to the innovative nature of COIL projects. Part of this attempt is through making the opportunities for professional learning through COIL known amongst university colleagues and administrators at home and elsewhere. As several instructors have pointed out, their involvement in COIL projects has drawn appreciation from colleagues and increased their reputation. One instructor credited the COIL projects with gains in their performance assessments, these initiatives counting as an internationalization initiative at their university:

"I'm acknowledged by a few people at my university as a COIL specialist, as I'm still the most experienced in this teaching strategy. A few more people in my department know that I do "different things" in class. Recently I was invited by the rectory to be part of a teaching innovation group, formed by 12 teachers from different departments" (Portuguese instructor).

\section{CONCLUSIONS}

COIL projects are time-consuming. They require instructors to acquire new skills as well as show understanding for the restrictions on as well as priorities of their colleagues. At the same time, the challenges that these instructors faced when engaging in COIL projects contributed to their professional development. As one instructor put it:

"The COIL project has been instrumental to my professional development as a lecturer and a researcher. It has provided a mechanism to ensure that the content, delivery and assessment of my modules are not only up-to-date with the latest experiential pedagogical thinking, but also provides students with future-proofed employability skills in the areas of intercultural and digital communication. The project has provided me with a high impact area of research which links directly to university and 
employer strategic priorities. By embedding the project in my Public Relations Theory \& Practice class I am able to give students an inter-disciplinary, cross-cultural experience within the broader business management subject area. The research informs the development of my teaching practice and the teaching provides a fresh source of research data each year to work with - a genuine win-win scenario for an academic with very little time to spare!" (UK instructor).

Articulating one's own pedagogics to another's diverging set of values, teaching alongside other colleagues, sharing materials and knowledge, and agreeing on standards and grading rubrics, the authors had to leave their comfort zones. By flipping the classroom and empowering students - some of who are not one's own - as well as colleagues to take command of the course content and learning outcomes, the instructors gave up control and embraced the ambiguity of the projects. Once the instructors were able to do exactly that, they could sit back and enjoy the fun. One instructor described why they continued to take on the additional work of COIL projects:

"My motivation: I have fun. It has to do with my own nature, as a teacher and as an individual. It makes me happy. It makes me a better teacher. I learn a lot. I expected to learn less and less, but actually it is more the other way around ... So, although it means tons of additional work (and it does not get better from project to project, as we are always looking for new ways to approach it and to increase the reach!!), I'm always looking forward to the next project" (Portuguese instructor).

The importance for educators to have fun, to spark creativity and explore different avenues in academic disciplines and pedagogy should not be underestimated. Institutions that encourage innovative approaches to teaching and learning do not only attract good students but also excellent instructors as well as interested community members. According to one instructor:

"My university is located in one of the most heavily concentrated areas of the US for global companies. As I talked about this project in the business community, I found myself invited to serve as the representative for higher education and international business for several organizations, including meeting with the Representative from the EU to the United States. These positions in turn have helped me grow as well as informed me of current business needs in global workplaces. Plus, I have enjoy working with the other instructors. Having someone-and in this case three someoneswho know exactly what you hope to achieve in the classroom is very encouraging" (US instructor).

Furthermore, institutional support of collaborative projects motivates research. As one instructor explained:

"I considered myself more a practitioner than an academic before I began collaborating with my other colleagues on COIL projects. One of us was interested in gathering data and publishing the results, which sparked my interest. Before I knew it, I was collaborating on several articles at once, publishing in peer reviewed journals and enjoying every bit of it" (Instructor in Germany).

It is, therefore, essential that institutions of higher learning encourage their instructors to engage in innovative projects such as COIL through policies which recognize their value for both the administration, the students and the instructors. These policies would include incentivizing instructors to engage in COIL projects through awards and reduced workloads, providing financial and personnel resources for instructors as well as investing in their IT infrastructure and professional development training, among many other examples [9]. In return, institutions would attract and retain motivated instructors, whose teaching and research contribute to the institutions' reputation and success.

\section{REFERENCES}

[1] D. Starke-Meyerring. "Designing globally networked learning environments: visionary pedagogies, partnerships, and policies." Keynote speech at SUNY Center for Online Collaborative Learning (COIL). Purchase, NY., October 19, 2007.

[2] D. Starke-Meyerring. "Globally networked learning environments in professional communication: challenging normalized ways of learning, teaching and knowing." Journal of Business and Technical Communication, vol. 24, pp. 259-266, 2010. https//doi.org/10.1177/ 1050651910363266

[3] B. Leask \& C. Bridge. "Comparing internationalisation of the curriculum in action across disciplines: Theoretical and practical perspectives." A Journal of Comparative and International Education, vol. 43, p. 80, 2013.

[4] UNESCO. "Global Flow of Tertiary-level Students." 2014. Retrieved from

http://www.uis.unesco.org/Education/Pages/international-student-flow-viz.aspx 
[5] N. Harrison. "Practice, problems and power in 'internationalisation at home': critical reflections on recent research evidence." Teaching in Higher Education, vol. 20, pp. 412-430. 2015. https//doi.org/10.1080/13562517.2015.1022147

[6] B. Leask \& J. Carroll. "Moving beyond 'wishing and hoping': Internationalisation and student experiences of inclusion and engagement." Higher Education Research \& Development, vol. 30, pp. 647-659, 2011.

[7] B. Leask. "Using formal and informal curricula to improve interactions between home and international students." Journal of Studies in International Education, vol. 13, pp. 205-211, 2009.

[8] C. Dunne. "Developing an intercultural curriculum within the context of the internationalisation of higher education: Terminology, typologies and power." Higher Education Research \& Development, vol. 30, pp. 609-622, 2011.

[9] M. Agnew \& H. Kahn. "Internationalization-at-Home: Grounded practices to promote intercultural, international, and global learning." Working with Diverse Communities, vol. 25, pp. 31-46, 2014. Retrieved from https://files.eric.ed.gov/fulltext/EJ1092773.pdf

[10] S. L. Butler. "Customizing college for a new generation." Career News, pp.1-3, October, 2018. Retrieved from https://www.higheredjobs.com/Articles/articleDisplay.cfm?ID=1750 \&utm_source=11_07_2018\&utm_medium=email\&utm_campaign=InsiderUpdate

[11] D. Starke-Meyerring \& D. Andrews. "Building a shared virtual learning culture." Business Communication Quarterly, 69, p. 33. Association for Business Communication, March 2006. https//doi.org/10.1177/1080569905285543 